\title{
IL-10 to TNF alpha ratios throughout early first trimester can discriminate healthy pregnancies from pregnancy losses
}

\section{Kaislasuo, Janina}

2020-01

Kaislasuo , J , Simpson , S , Petersen , J F , Peng , G , Aldo , P , Lokkegaard, E, Paidas , M , Pal , L , Guller , S \& Mor , G 2020 , ' IL-10 to TNF alpha ratios throughout early first trimester can discriminate healthy pregnancies from pregnancy losses ' , American Journal of Reproductive Immunology , vol. 83 , no. 1 , 13195 . https://doi.org/10.1111/aji.13195

http://hdl.handle.net/10138/319803

https://doi.org/10.1111/aji.13195

unspecified

acceptedVersion

Downloaded from Helda, University of Helsinki institutional repository.

This is an electronic reprint of the original article.

This reprint may differ from the original in pagination and typographic detail.

Please cite the original version. 
DR SETH GULLER (Orcid ID : 0000-0003-0651-8241)

PROFESSOR GIL MOR (Orcid ID : 0000-0002-5499-3912)

Article type : Short Communication

Corresponding author mail-id: gil.mor@yale.edu

IL-10 to TNF a ratios throughout early first trimester can discriminate healthy pregnancies from pregnancy losses

Janina Kaislasuo*1,2, Samantha Simpson¹, Jesper F Petersen³, Gang Peng ${ }^{4}$, Paulomi Aldo¹, Ellen Lokkegaard $^{3}$, Michale Paidas ${ }^{5}$, Lubna Pal ${ }^{1}$, Seth Guller ${ }^{1}$, Gil Mor ${ }^{1,6}$

${ }^{1}$ Department of Obstetrics, Gynecology and Reproductive Sciences, Division of Reproductive Sciences, Yale School of Medicine, New Haven, CT, USA. ${ }^{2}$ Department of Obstetrics and Gynecology, University of Helsinki and the Helsinki University Hospital, Helsinki, Finland. ${ }^{3}$ Department of Obstetrics and Gynecology, North Zealand Hospital, Hilleroed, Denmark. ${ }^{4}$ Department of Biostatistics, School of Public Health, Yale University, New Haven, CT, USA. ${ }^{5}$ Department of Obstetrics, Gynecology and Reproductive Sciences, University of Miami Miller School of Medicine, Miami, Fl, USA. ${ }^{6}$ C.S. Mott Center for Human Growth and Development, Department of Obstetrics, Gynecology, Wayne State University, Detroit, MI, USA.

${ }^{*}$ Kaislasuo and Simpson should be considered joint first author

This article has been accepted for publication and undergone full peer review but has not been through the copyediting, typesetting, pagination and proofreading process, which may lead to differences between this version and the Version of Record. Please cite this article as doi: 10.1111/aji.13195

This article is protected by copyright. All rights reserved 
Acknowledgments The authors thank A Cunha and D Leonard for assistance with patient recruitment and sample collection, and $\mathrm{J}$ Shaw and $\mathrm{R}$ Cortes and S Nichols-Burns for assistance with sample processing and storage (Yale University Fertility Center).

Key words (3-8): pregnancy loss, embryo implantation, TNFa, interleukin-10, inflammation, infertility.

\begin{abstract}
Problem: Embryo implantation and placentation require a careful immunological balance. Cytokines such as IL-10 and TNFa have been implicated as markers of dysregulation, but have only been studied at a single time point or after a pregnancy lost. Our objective was to determine normative patterns of serum levels of IL-10 and TNFa and their ratio throughout the first trimester in healthy pregnancies, and to determine if this pattern differs from pregnancy loss.
\end{abstract}

Methods: Two prospective longitudinal cohorts of gravidae including in vitro fertilization (IVF) and naturally conceived pregnancies with serial blood draws. Cytokines were assayed using SimplePlex. In the IVF cohort we monitored from the implantation day up to 6 weeks of gestation; whereas in the naturally conceived cohort, sample collection began at 4 weeks and throughout the whole first trimester.

Results: IL-10 concentrations in normal pregnancies were significantly higher than in pregnancies ending in a loss starting at 6-8 weeks of gestation while TNFa concentrations were significantly lower in normal than in pregnancies ending in a loss starting at 3-5 of gestation weeks. The IL-10 to TNFa ratio in normal pregnancies was significantly higher from 4 to 9 weeks compared to pregnancies that were lost (t-test, $p<0.05)$. Changes were observed before any symptoms of miscarriage were present.

This article is protected by copyright. All rights reserved 
Conclusions: We provide evidences of differences in early immunomodulation in healthy pregnancies versus those destined to end in first trimester loss. The ratio of IL-10 to TNFa rises significantly higher in viable pregnancies as early as 4.5 weeks compared to pregnancies loss.

\section{STUDY FUNDING/COMPETING INTEREST(S)}

The authors received no specific funding for this work and disclose no conflicts of interest

\section{TRIAL REGISTRATION NUMBER}

N/A

This article is protected by copyright. All rights reserved 


\section{Introduction}

The implantation of a semi-allogenic embryo and its ongoing development requires careful checks and balances in the maternal immune system. Cellular and humoral immunity are an ongoing area of study owing to their critical role in normal pregnancy. Alteration of cellular and humoral immunity might be associated with pregnancy complications, specifically recurrent implantation failure (RIF) and recurrent pregnancy loss (RPL) $)^{1,2}$. Immunologic explanations have also been implicated as an important reason behind early pregnancy loss in women without this history ${ }^{3}$.

Cytokines and chemokines are a family of secreted immune modulators controlling the function and differentiation of both immune and non-immune cells. During pregnancy, cytokines and chemokines have been shown to be critical for the success of implantation, trophoblast invasion, and immune regulation of the maternal immune system ${ }^{4-6}$. At five days after fertilization, the process of implantation depends on the preparation of the uterine surface epithelium to allow a dialogue with and subsequent attachment of the embryonic trophectoderm. The preparation of the epithelium requires an inflammatory process that removes the mucin layer covering the epithelium, and the exposure of adhesion molecules that facilitate the attachment of the embryo ${ }^{7,8}$. After attachment, the blastocyst begins migration into the underlying decidua. This process is tightly regulated by immune cells present in the decidua, which secrete pro-inflammatory cytokines and chemokines to attract the

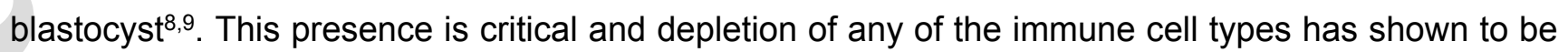
detrimental, resulting in fetal loss or impaired growth in animal studies ${ }^{10}$.

Excessive inflammation can also be detrimental ${ }^{1}$. Therefore, the success of implantation and pregnancy in general depends on the maintenance of a balance between pro-and anti-inflammatory signals. This balance is defined as the ratio of pro- and anti-inflammatory cytokines, and is a process regulated by both the maternal local environment and the implanting blastocyst. The blastocyst secrets immunomodulatory factors to dampen inflammation and gradually switch the environment to an anti-inflammatory profile as placentation is established ${ }^{9,11}$.

Various cytokines have been examined as markers of immune dysregulation in early pregnancy loss, with findings of prolonged upregulation of T-helper 1(Th1) mediated proinflammatory responses compared to T-helper 2 (Th2) mediated anti-inflammatory responses being associated with complications ${ }^{3,12-14}$. Anti-inflammatory Interleukin-10 (IL-10) and pro-inflammatory Tumor Necrosis Factor alpha (TNFa), both key cytokines in the cascade of immune signaling, have

This article is protected by copyright. All rights reserved 
been identified as potential markers of immune dysregulation in studies on pregnant women ${ }^{15-23}$. Unfortunately, the results from studies to date are inconsistent and contradictory, making it difficult to interpret the clinical relevance of the published data.

Most of the reported studies have evaluated the inflammatory status at only one inconsistent timepoint in the first trimester and have used either nonpregnant controls as a reference, or compared normal pregnancies to confirmed miscarriages. In these studies, women with normal pregnancies show a more anti-inflammatory profile compared both to nonpregnant women and those with a diagnosed miscarriage ${ }^{13,15,16,18-20}$. These results do not clearly establish if the more proinflammatory profile seen in diagnosed miscarriages develops before a loss is diagnosed, or if it is simply reflects that the immune system has returned to a normal state. Additionally, since pregnancy is an evolving process, the evaluation at a single time point only provides a snapshot limited to the specific period when the sample was taken. There is growing evidence that pregnancy represents physiologically distinct immunological conditions and time periods ${ }^{9}$. Therefore, looking at one single time cannot provide adequate information on this delicate evolving process. A longitudinal monitoring of cytokine profiles has not been standardized for gestational age. Establishing this is critical since implantation, trophoblast invasion, and placental/fetal development are active processes that requires a continuous adaptation of the environment.

A major limitation on the potential clinical application of circulating cytokines for the prediction of pregnancy complication is the lack of standardization. The methodology used for the evaluation of circulating cytokines in prior studies varies between traditional ELISAs and multiplexing platforms such as Luminex. The reproducibility of results between prior studies is poor, with both highly varying numeric results from each of these platforms and even opposite results in different studies, thus making it difficult to apply their findings to clinical use. Consequently, there is a need to implement a platform that is simple and reproducible, allowing the standardization of the normal levels of circulating cytokines. Simple Plex ${ }^{\mathrm{TM}}$ platform is a novel multi-analyte, automated microfluidic platform that allows the evaluation of cytokines and chemokines from human biological samples ${ }^{24}$. In previous reported studies, Simple Plex ${ }^{\mathrm{TM}}$ has shown significant advantages over traditional approaches in terms of low sample volume requirements, sensitivity and dynamic range, coefficient of variation and reproducibility; characteristics indispensable for the establishment of a diagnostic test with potential clinical applications ${ }^{24,25}$.

In the current study, we established a platform, Simple Plex ${ }^{T M}$, to evaluate serum cytokine levels in a clinical setting, demonstrating the utility of an automated, fast, simple, sensitive and 
reproducible cytokine test. We standardized and measured the concentrations of two recognized markers of immune dysregulation, IL-10 and TNFa, throughout the whole first trimester, and demonstrated that the levels of the individual cytokines and the IL-10:TNFa ratio develops differently in normal pregnancies compared to pregnancies that are subsequently lost in the first trimester.

\section{Materials and Methods}

\section{Study population}

This study includes 506 serum samples collected between 3 to 13 weeks (25 to 96 days) of gestation. To be able to include enough samples from the earliest timepoints in pregnancy, samples were collected from two prospectively followed pregnancy cohorts - women undergoing in vitro fertilization (IVF) treatment $(n=40)$ and women with natural conceptions $(n=102)$. A total of 97 women carried an uncomplicated full-term pregnancy (controls) and 45 women experienced a first trimester pregnancy loss (losses, Table 2). Samples from the IVF cohort included 83 data points for controls (2-6 data points for each individual) and 85 for losses (2-7 data points for each individual). The natural conception cohort included 270 data points for controls (3-4 data points for each individual) and 68 for losses (2-4 data points for each individual).

A limitation of this cohort is the lack of racial diversity. Due to the country of enrollment (Denmark) the majority of the patients are Caucasian ${ }^{26}$.

\section{Patient recruitment and sample collection}

IVF Cohort. Recruitment of IVF patients and storage of samples was approved by the Yale institutional IRB with no written consent requirement (\#2000021607). Patients aged 18-44 undergoing fresh or frozen day 3 or day 5 (blastocyst) embryo transfer from October 2017 to July 2018 were eligible for participation. Exclusion criteria were patients with chronic autoimmune disease (such as lupus, thyroid antibodies, ulcerative colitis, or Crohn's disease), diabetes and hypertension requiring medication, endometriosis confirmed by laparoscopy, or current illness (in general, we excluded patients with underlying inflammatory process). We also excluded patients with prior losses, unless the tissue from the loss had undergone genetic testing and was determined to be chromosomally abnormal. Patients were asked to participate at the time of embryo transfer. Blood was collected by venipuncture into $10 \mathrm{~mL}$ vacutainer tubes starting at the time of the first positive $\beta$ hcg, 8-12 days after embryo transfer and then every 48 hours until an intrauterine pregnancy was

This article is protected by copyright. All rights reserved 
confirmed using transvaginal ultrasound, or when a pregnancy was deemed as biochemical based on declining $\beta$-hcg levels following an initial positive test. Samples were left at room temperature for 60 minutes to allow for clotting and then centrifuged (Thermo Scientific Sorvall ST 16, Waltham, MA) at 3,000 RPM for ten minutes at room temperature. Serum was aliquoted into $1.5 \mathrm{~mL}$ polypropylene RNase- and DNase-free microcentrifuge tubes and stored in $-80^{\circ} \mathrm{C}$ freezers until ready for testing.

Naturally Conceived Cohort. The natural conception cohort was part of a larger Danish prospective early pregnancy cohort (the PEP cohort) recruiting women through online ads in $2016-201726$. Healthy women with a singleton pregnancy, able to understand and sign written consent, and aged at least 18 years were eligible for participation. Exclusion criteria included history of recurrent pregnancy loss ( $\geq 3$ losses, including biochemical pregnancies), any type of assisted reproductive techniques, uterine or tubal abnormalities assessed at the first visit, and ongoing substance abuse. Follow-up with serial blood draws and transvaginal ultrasound started as soon as the women expressed interest after a positive urine pregnancy test and continued every two weeks until completion of the first trimester (11-14 weeks of gestation) or a diagnosed pregnancy loss. Blood was collected by venipuncture into vacutainer separator tubes (BD Diagnostics, Franklin Lakes, NJ, USA), allowed to clot for 15 minutes at room temperature and then centrifuged (Hettich Rotina 380 R, Andreas Hettich $\mathrm{GmbH}$, Tuttlingen, Germany) for $10 \mathrm{~min}$ at $3500 \mathrm{rpm}$ at $5^{\circ} \mathrm{C}$. Serum was aliquoted into plastic vials equal to the IVF cohort and stored in $-80^{\circ} \mathrm{C}$ freezers until ready for testing.

All specimens from both cohorts were transported on liquid nitrogen to Yale University.

\section{Simple Plex ${ }^{T M}$ Immunoassay}

All samples were tested using the Simple Plex ${ }^{\mathrm{TM}}$ platform (ProteinSimple, San Jose, CA), an automated cartridge-based immunoassay with microfluidic technology 24 . The cartridge is premade by the manufacturer according to individual needs with one to four analytes. Within the cartridge all the steps of a sandwich ELISA are executed automatically, thus excluding human errors, and allowing for fast and sensitive results. Samples are separated into parallel channels if analyzing multiple analytes and each analyte is analyzed in triplicate glass nanoreactors, eliminating cross-reactivity when multiplexing and generating a triplicate reading of each analyte. Sample concentrations $(\mathrm{pg} / \mathrm{mL})$ are automatically calculated by fitting RFUs to calibration curve parameters provided with each cartridge and adjusted for possible user defined dilution factors. The platform has been characterized and validated in a previously reported study ${ }^{24}$ and showed superior performance in a study comparing multiple platforms ${ }^{25}$.

This article is protected by copyright. All rights reserved 
Serum samples were diluted 1:2 with diluent provided by the manufacturer and $50 \mu$ l of diluted sample was loaded into the cartridge. The performance of the platform revealed high reproducibility and time efficiency, with the whole process taking only 80 minutes. The data was exported to Microsoft Excel for analysis. The lower limits of detection were $0.46 \mathrm{pg} / \mathrm{ml}$ for IL-10 and $1.14 \mathrm{pg} / \mathrm{ml}$ for TNFa. The intra- and inter-assay coefficients of variance were $2.1-4.9 \%$ and $4.3 \%$ for IL-10 and $3.3-10.4 \%$ and $6.9 \%$ for TNFa.

\section{Statistical analysis}

The sample size was determined based on the risk of miscarriage, which is $15 \%$. The original design of natural conceptions cohort had the objective to enroll 30 women who miscarried 170 uncomplicated controls. With this sample size we can get $95 \%$ power and $5 \%$ alpha error level in an unpaired two-tailed test with Cohen's D around 0.75 . Even though we only collected 27 women who miscarried and 75 controls, we can get $90 \%$ power in the same test. A limitation of natural pregnancy cohort is that the data is usually collected after 5-7 weeks of pregnancy. We included IVF cohort data to get more information at early pregnancy stage and also improve the statistical power. Before the statistical analysis, we removed 3 outliers with IL-10 larger than $20 \mathrm{pg} / \mathrm{ml}$ and 2 outliers with TNFa larger than $15 \mathrm{pg} / \mathrm{ml}$. We compared differences between controls and losses at different phases of placentation based on gestational age using ANOVA. We then used the Locally Weighted Scatterplot Smoothing (LOWESS) method to estimate the changes of IL-10, TNFa, and the ratio of IL-10 to TNF $\alpha$ across gestational age for controls and losses separately. Within this model, we then estimated the difference of the individual cytokines and their ratio between controls and losses using Cohen's D and t-test within each week. A positive Cohen's D indicates that controls have higher levels than the losses, while a negative Cohen's $D$ indicates the controls have lower levels than the losses. Unlike p-value, the sample size has no influence on the statistical significance of Cohen's D.

Statistical analyses and graphs were done in R software 3.5.1 and GraphPad Prism v8

(GraphPad Software Inc., San Diego, CA). A p-value less than 0.05 was considered statistically significant.

\section{Results}

Variations on the measurement of cytokines during pregnancy

We looked at previous reports evaluating circulating IL-10, TNFa, and their ratio in human serum in early pregnancy and found 13 studies that met our inclusion criteria of comparing normal ongoing 
pregnancies to miscarriages in the first trimester. These studies consist of the evaluation of only one sample from differing phases of pregnancy (Table 1). Overall, the reported values of IL-10 and TNF $\alpha$ in these studies are extremely variable, even with the same method used for analyzing samples. In general, IL-10 levels were found to be lower in women who went on to experience a loss when compared to normal pregnancies (Table 1). For TNFa, there is no clear pattern between the different studies. Only one study looked at the ratio of the cytokines, concluding that the profile was shifted toward a pro-inflammatory profile before a miscarriage happened ${ }^{16}$. These results exemplify the need for standardization of the methodology for evaluating circulating cytokines and the timing when the samples are evaluated.

\section{Serum levels of IL-10 and TNFa normal pregnancies and first trimester losses}

To achieve our objective of delineating the cytokine profile of pregnant women throughout the first trimester, we combined two cohorts: an IVF cohort where we were able to monitor the day of implantation and collect samples from the earliest stages of pregnancy (between 3 and 6 weeks of gestation), and a naturally conceived cohort, where sample collection began at 4 weeks and continued throughout the whole first trimester. Demographic information about the cohorts is given in Table 2. The IVF cohort was significantly older than the naturally conceived cohort, but otherwise both cohorts were similar in BMI, previous live birth, previous miscarriage, and race/ethnicity.

To test whether there is a correlation between the levels of circulating levels of IL-10 and TNFa throughout fetoplacental development and if this differs in normal pregnancy and pregnancies ending in first trimester loss, we measured the concentrations of IL-10 and TNFa in serum of these women and evaluated the cytokine expression pattern during established biological phases of placentation ${ }^{27}$. Samples were grouped into peri-implantation phase (3-5 weeks), histotrophic phase (6-8 weeks), and perfusion phase (9-13 weeks), based on gestational age at blood draw (Figure 1 and Table 3). In patients with normal pregnancies, IL-10 at peri-implantation had a mean of $2.5 \mathrm{pg} / \mathrm{mL}$, which increased to $2.7 \mathrm{pg} / \mathrm{mL}$ in the histotrophic phase and $2.8 \mathrm{pg} / \mathrm{mL}$ in the perfusion phase (Fig. $1 \mathrm{~A}$ and Table 3). TNFa in normal pregnancies started at an average of $5.8 \mathrm{pg} / \mathrm{mL}$ in the peri-implantation phase and decreased to $5.4 \mathrm{pg} / \mathrm{mL}$ in the histotrophic phase, staying steady in the perfusion phase at $5.5 \mathrm{pg} / \mathrm{mL}$ (Fig. 1B). In pregnancies that resulted in a loss, the IL-10 concentrations started at a lower level, at $2.3 \mathrm{pg} / \mathrm{mL}$, and instead of increasing, stayed at this level in the histotrophic phase, a significant difference compared to the levels in normal pregnancies ( $p \leq 0.01$, Fig $1 \mathrm{~A}$ and Table 3 ). TNFa concentrations in pregnancies that resulted in a loss started significantly higher at $6.6 \mathrm{pg} / \mathrm{mL}$ in

This article is protected by copyright. All rights reserved 
the peri-implantation phase, and remained higher in the histotrophic phase at $6.2 \mathrm{pg} / \mathrm{mL}$ compared to the normal pregnancies ( $p \leq 0.01$, Fig 1B and Table 3).

\section{The IL-10:TNFa ratio in normal pregnancies and first trimester losses}

After determining the concentrations for IL-10 and TNF $\alpha$ individually during early pregnancy, we evaluated whether the ratio of IL-10:TNF $\alpha$ could provide better information on the balance of inflammation taking place during early pregnancy. In normal pregnancies, the ratio of IL-10:TNFa started at an average of 0.45 at peri-implantation, increased to 0.53 during the histotrophic phase, and remained steady at this level in the perfusion phase (Fig. 1C and Table 3). In patients with pregnancies resulting in a loss, we observed significant differences in the IL-10:TNF $\alpha$ ratio compared to normal pregnancies. The ratio started significantly lower at peri-implantation $(0.37, p=0.01)$, and remained significantly lower in the histotrophic phase $(0.38, p=0.001)$ (Fig $1 \mathrm{C}$ and Table 3 ), whereas we were unable to evaluate the differences in the perfusion phase as we had to few samples from losses in that phase.

\section{Anti-/Pro- inflammatory model for normal pregnancies and first trimester losses}

We then developed a statistical model to determine whether normal fetoplacental function could be predicted based on the pattern of expression of these two cytokines or their ratio. As shown in Fig. 2, we observed that in controls, IL-10 starts at a somewhat lower level at 4 weeks gestation and steadily increases until week 8 (Fig. 2A). This pattern differed in loses where, although starting at a similar level, the IL-10 level decreased dramatically during the first weeks and never returned to the initial normal level thereafter (Fig 2A). In controls TNFa decreased from week 4 through 8 and then remained steady thereafter (Fig 2B). In losses the TNFa profile was again opposite to that in controls. Although starting at almost the same level as the controls, the TNFa continued to increase until 6-7 weeks, and remained higher than in controls for the rest of the evaluated gestational weeks. The differences in expression between controls and losses became clearer when looking at the ratio of IL-10:TNFa (Fig 2C and Table 4), with a statistically significant separation starting at week 5 $(p<0.001)$. In controls, the IL-10:TNF $\alpha$ ratio continued to rise from implantation until 8 weeks, while in losses the ratio behaved opposite, decreasing starting at implantation.

When comparing IVF pregnancies and natural conception, we did not see any significant differences between the cohorts when comparing samples taken at similar gestational age for either the individual cytokines or their ratio (Fig. 3).

This article is protected by copyright. All rights reserved 


\section{Discussion}

In the present study, we describe normative longitudinal serum concentrations for IL-10 and TNFa in early pregnancy and for the first time illustrate how the IL-10:TNF $\alpha$ ratio develops throughout the whole first trimester and how this profile differs in pregnancies destined for loss, before a diagnosis or any symptoms.

Normal pregnancy shows an early pro-inflammatory profile followed by a clear shift towards an anti-inflammatory profile immediately after implantation, characterized by an increase in IL-10, decrease in TNFa, and increasing IL-10:TNFa ratio. Interestingly, pregnancy loss was associated with a failure in this shift, with no increase in neither IL-10 alone nor in the IL-10:TNFa ratio. These findings provide evidence in support for the relevance of the shift from inflammation to antiinflammation necessary for the maintenance of the pregnancy ${ }^{9}$ and support earlier findings defining normal pregnancy as an anti-inflammatory condition ${ }^{27,28}$. Furthermore, our findings for the first time illustrate that the shift happens very early, right after implantation.

In both normal pregnancies and losses, the TNFa level was highest at early implantation, which is in line with prior evidence of a pro-inflammatory profile supporting implantation ${ }^{5,8,10}$. In pregnancies ending in a loss TNFa was higher than in normal pregnancies at the earliest phase (Figure 1), possibly reflecting either excessive inflammation or a disturbed local immune education leading to the failure to shift the balance towards a more anti-inflammatory profile reflected by the rising IL-10:TNFa ratio seen in normal pregnancies.

Prior studies looking at IL-10 and TNFa as predictive biomarkers contribute to the knowledge of immune balance, but studies are small and conclusions are based on single samples taken at differing timepoints during the first trimester, with inconclusive results (Table 1). IL-10 has previously been shown to be comparatively lower and TNFa comparatively higher in patients who have just been diagnosed with miscarriage ${ }^{17}$ and in patients with threatened miscarriage who go on to miscarry ${ }^{18}$ compared to normal pregnancies. In at least two studies, it has been shown that in both patients with and without a history of RPL, IL-10 is higher in pregnancies that go on to have live births compared to pregnancies that don't15,19. Conversely, one study found that the IL-10 concentration is significantly lower in pregnancies that go on to have live births ${ }^{20}$. In patients who had undergone IVF, TNFa levels in the 5th week of gestation were significantly higher in women who went on to have a pregnancy loss ${ }^{21}$ and also in women who had already miscarried, TNFa levels

This article is protected by copyright. All rights reserved 
were elevated when compared to women with healthy pregnancies ${ }^{22}$. Alternately, one study found no differences in either IL-10 or TNF $\alpha$ concentrations between patients in the first trimester who went on to have a healthy pregnancy versus those who had a miscarriage ${ }^{23}$. A meta-analysis comparing RPL patients to patients with normal pregnancy found that TNFa was higher in the recurrent pregnancy loss patients, but not all studies included in the meta-analysis looked at pregnant patients, sample timing was inconsistent between evaluated studies and some studies evaluated the RPL patients after a diagnosed loss, not before ${ }^{16}$. These studies, looking at only one time point and with varying methodologies and definitions of controls and cases do not clearly delineate the distinct immunological time periods represented by the first trimester.

The process of implantation and placentation is a continuum of crosstalk between the maternal and fetal side. At five days after fertilization, it is known that the epithelium must demonstrate receptivity to the invading trophoblast by secreting pro-inflammatory cytokines and protease inhibitors ${ }^{10,11,29}$, and in response the trophoblast indirectly stimulates Th2 cytokine production ${ }^{3}$. Up to 4.5 weeks gestation, the crosstalk between the embryo and the maternal uterine environment is suspected largely to focus on this invasion. This correlates with our finding that in normal pregnancy at this time, the serum IL-10:TNFa ratio is balanced and similar in pregnancies that are destined to miscarry. In our study, miscarriages have an unchanged IL-10:TNFa ratio at 5 weeks, whereas normal pregnancies have an increasing ratio. The profiles take a different direction from hereon. By 6 weeks, implantation is completed and the embryo relies primarily on glandular nutrition while the process of vascularization begins, a process requiring continued migration of cells and modulation of the environment. By 9 weeks the early blood supply is established and along with that oxidative tension gradually increases, yet again posing different challenges for the embryo ${ }^{30,31}$. Since the mechanism of trophoblast invasion and blood vessel recruitment is not clearly understood but is thought to rely at least partially on cytokine signaling, delineating the timing of cytokine aberrations in these different phases in both patients with normal pregnancy and early miscarriage is important.

Our study improves upon the foundation established by past research by following longitudinal changes in these serum biomarker concentrations throughout the first trimester, and enrolling subjects prior to any signs or symptoms of miscarriage. By utilizing IVF patients, we were able to obtain the earliest information possible. To our knowledge this is also the first study comparing IVF pregnancies and natural conceptions and we did not see any significant differences

This article is protected by copyright. All rights reserved 
between the cohorts when comparing samples taken at similar gestational age for either the individual cytokines or their ratio (Fig. 3).

The platform used to analyze the samples displayed a high sensitivity, with readings from all 506 samples being within the detection rate and with low variability. The fully automatic design enabled fast analysis and excluded human errors.

A clear weakness of the study was the lack of samples before conception. We were unable to assess how the cytokine levels and ratio change after conception. Also, as most losses were diagnosed by week 8 , our samples size in the losses group from week 9 onwards is insufficient in demonstrating significant differences between controls and losses in late first trimester, although the trends were similar. Furthermore, we only had access to histologic evaluation or genetic testing 5/45 losses $(11 \%)$, and therefore cannot be certain that all losses were chromosomally normal. This may be of importance as others have shown differences in inflammation between losses with normal and abnormal karyotype ${ }^{32}$.

Much of prior studies have focused on immunological differences between healthy women and women with repeated pregnancy losses or in a population where miscarriage has already been diagnosed ${ }^{15,33,34}$. Here we show that the immunological profile differs in healthy women with subsequent pregnancy loss compared to normal pregnancies, before any symptoms. Standardizing and demonstrating this difference longitudinally in first trimester serum samples from seemingly healthy women fuels further research and strives towards finding immunologic predictive tests.

This article is protected by copyright. All rights reserved 


\section{Acknowledgements}

The authors wish to thank the clinical and laboratory team of the Department of Obstetrics Gynecology and Reproductive Sciences at Yale University. We also would like to thank the Joey Guy for reviewing the manuscript.

\section{Authors' roles}

G.M., L.P. and M.P. designed the study and interpreted the results. K.K. and S.S performed data analysis and wrote the article. G.P. performed statistical analysis and model design. P.A. performed cytokine assays. S.G, JFP and EL contributes with patient's enrollment, sample collection and data collection. All authors participated in data analysis and critical reading and editing of the article. All authors approved the final version of the article.

\section{Funding}

No funding was obtained.

\section{Conflict of interest}

None declared

This article is protected by copyright. All rights reserved 


\section{References}

1. Krieg S, Westphal L. Immune Function and Recurrent Pregnancy Loss. Semin Reprod Med. 2015;33(4):305-312.

2. Shetty S, Ghosh K. Anti-phospholipid antibodies and other immunological causes of recurrent foetal loss--a review of literature of various therapeutic protocols. Am J Reprod Immunol. 2009;62(1):9-24.

3. Kwak-Kim J, Bao S, Lee SK, Kim JW, Gilman-Sachs A. Immunological modes of pregnancy loss: inflammation, immune effectors, and stress. Am J Reprod Immunol. 2014;72(2):129-140.

4. Raghupathy R, Makhseed M, Azizieh F, Omu A, Gupta M, Farhat R. Cytokine production by maternal lymphocytes during normal human pregnancy and in unexplained recurrent spontaneous abortion. Hum Reprod. 2000;15(3):713-718.

5. Boomsma CM, Kavelaars A, Eijkemans MJ, et al. Endometrial secretion analysis identifies a cytokine profile predictive of pregnancy in IVF. Hum Reprod. 2009;24(6):1427-1435.

6. Ng SC, Gilman-Sachs A, Thaker P, Beaman KD, Beer AE, Kwak-Kim J. Expression of intracellular Th1 and Th2 cytokines in women with recurrent spontaneous abortion, implantation failures after IVF/ET or normal pregnancy. Am J Reprod Immunol. 2002;48(2):77-86.

7. Dekel N, Gnainsky Y, Granot I, Mor G. Inflammation and implantation. Am J Reprod Immunol. 2010;63(1):17-21.

8. Dekel N, Gnainsky Y, Granot I, Racicot K, Mor G. The role of inflammation for a successful implantation. Am J Reprod Immunol. 2014;72(2):141-147.

9. Mor G, Aldo P, Alvero AB. The unique immunological and microbial aspects of pregnancy. Nat Rev Immunol. 2017;17(8):469-482.

10. Mor G, Cardenas I, Abrahams V, Guller S. Inflammation and pregnancy: the role of the immune system at the implantation site. Ann N Y Acad Sci. 2011;1221:80-87.

11. Mor G, Cardenas I. The immune system in pregnancy: a unique complexity. Am J Reprod Immunol. 2010;63(6):425-433.

12. Tripathi S, Guleria I. Role of PD1/PDL1 pathway, and TH17 and treg cells in maternal tolerance to the fetus. Biomed J. 2015;38(1):25-31.

13. Saito S, Nakashima A, Shima T, Ito M. Th1/Th2/Th17 and regulatory T-cell paradigm in pregnancy. Am J Reprod Immunol. 2010;63(6):601-610.

This article is protected by copyright. All rights reserved 
14. Makrigiannakis A, Petsas G, Toth B, Relakis K, Jeschke U. Recent advances in understanding immunology of reproductive failure. J Reprod Immunol. 2011;90(1):96-104.

15. Jenkins C, Roberts J, Wilson R, MacLean MA, Shilito J, Walker JJ. Evidence of a $T(H) 1$ type response associated with recurrent miscarriage. Fertil Steril. 2000;73(6):1206-1208.

16. Zhang C, Deng X, Zhang X, et al. Association between Serum TNF-alpha Levels and Recurrent Spontaneous Miscarriage: A Meta-analysis. Am J Reprod Immunol. 2016;75(2):86-93.

17. Calleja-Agius J, Schembri-Wismayer P, Calleja N, Brincat M, Spiteri D. Obstetric outcome and cytokine levels in threatened miscarriage. Gynecol Endocrinol. 2011;27(2):121-127.

18. Calleja-Agius J, Muttukrishna S, Pizzey AR, Jauniaux E. Pro- and antiinflammatory cytokines in threatened miscarriages. Am J Obstet Gynecol. 2011;205(1):83 e88-16.

19. Paradisi R, Maldini-Casadei M, Boni P, Busacchi P, Porcu E, Venturoli S. T-helper 2-cytokine levels in women with threatened abortion. Eur J Obstet Gynecol Reprod Biol. 2003;111(1):43-49.

20. Vassiliadis S, Ranella A, Papadimitriou L, Makrygiannakis A, Athanassakis I. Serum levels of pro- and anti-inflammatory cytokines in non-pregnant women, during pregnancy, labour and abortion. Mediators Inflamm. 1998;7(2):69-72.

21. Freis A, Schlegel J, Daniel V, Jauckus J, Strowitzki T, Germeyer A. Cytokines in relation to hCG are significantly altered in asymptomatic women with miscarriage - a pilot study. Reprod Biol Endocrinol. 2018;16(1):93.

22. Verma P, Verma R, Nair RR, et al. Altered crosstalk of estradiol and progesterone with Myeloidderived suppressor cells and Th1/Th2 cytokines in early miscarriage is associated with early breakdown of maternal-fetal tolerance. Am J Reprod Immunol. 2019;81(2):e13081.

23. Barrientos G, Fuchs D, Schrocksnadel K, et al. Low levels of serum asymmetric antibodies as a marker of threatened pregnancy. J Reprod Immunol. 2009;79(2):201-210.

24. Aldo P, Marusov G, Svancara D, David J, Mor G. Simple Plex() : A Novel Multi-Analyte, Automated Microfluidic Immunoassay Platform for the Detection of Human and Mouse Cytokines and Chemokines. Am J Reprod Immunol. 2016;75(6):678-693.

25. Fischer SK, Carrasco-Triguero M, Hong K, et al. Commercial biomarker assays: friend and foe. Bioanalysis. 2016;8(22):2351-2362.

26. Friis Petersen J, Friis-Hansen LJ, Jensen AK, Nyboe Andersen A, Lokkegaard ECL. Early pregnancy reference intervals; 29 serum analytes from 4 to 12 weeks' gestation in naturally conceived and

This article is protected by copyright. All rights reserved 
uncomplicated pregnancies resulting in live births. Clinical chemistry and laboratory medicine : CCLM / FESCC. 2019.

27. Nicholson R, ebrary Inc. The placenta development, function and diseases. In: An overview ofdevelopment, functions and diseases of the placenta. Hauppauge, N.Y.: Nova Science Publishers, Inc.,; 2013: http://site.ebrary.com/lib/yale/Doc?id=10693655.

28. Hill JA, Polgar K, Anderson DJ. T-helper 1-type immunity to trophoblast in women with recurrent spontaneous abortion. JAMA. 1995;273(24):1933-1936.

29. Raghupathy R, Makhseed M, Azizieh F, Hassan N, Al-Azemi M, Al-Shamali E. Maternal Th1- and Th2type reactivity to placental antigens in normal human pregnancy and unexplained recurrent spontaneous abortions. Cell Immunol. 1999;196(2):122-130.

30. Roberts VHJ, Morgan TK, Bednarek P, et al. Early first trimester uteroplacental flow and the progressive disintegration of spiral artery plugs: new insights from contrast-enhanced ultrasound and tissue histopathology. Hum Reprod. 2017;32(12):2382-2393.

31. Burton GJ, Jauniaux E. Placental oxidative stress: from miscarriage to preeclampsia. J Soc Gynecol Investig. 2004;11(6):342-352.

32. Yamada H, Morikawa M, Furuta I, et al. Circulating cytokines during early pregnancy in women with recurrent spontaneous abortion: decreased TNF-alpha levels in abortion with normal chromosome karyotype. Hokkaido Igaku Zasshi. 2004;79(3):237-241.

33. Arslan E, Colakoglu M, Celik C, et al. Serum TNF-alpha, IL-6, lupus anticoagulant and anticardiolipin antibody in women with and without a past history of recurrent miscarriage. Arch Gynecol Obstet. 2004;270(4):227-229.

34. Calleja-Agius J, Jauniaux E, Muttukrishna S. Placental villous expression of TNFalpha and IL-10 and effect of oxygen tension in euploid early pregnancy failure. Am J Reprod Immunol. 2012;67(6):515525.

This article is protected by copyright. All rights reserved 


\section{Figure Legends.}

Figure 1. Levels of IL-10 (A), TNFa (B) and their ratio (C) shown according to biological phases of placentation. 4-5 weeks refers to the peri-implantation phase, 6-8 weeks to the histotrophic phase, and 9-13 weeks to the perfusion phase. Data presented as mean+/-SD. ${ }^{*} p \leq 0.01,{ }^{* *} p<0.001$

Figure 2. The changes of IL-10 (A), TNFa (B) and their ratio (C) across gestational age. The line represents the mean estimation by LOWESS and the colored areas the $95 \%$ confidence intervals of the mean estimation. Only the ratio is significantly different between the groups $(p<0.05)$

Figure 3. The changes of IL-10 (A), TNFa (B) and their ratio (C) in IVF pregnancies and natural conceptions. There is no difference between the cohorts in normal pregnancies. In IVF pregnancies ending in a loss both IL-10 and TNF $\alpha$ are higher than in natural conceptions ending in a loss, but the ratio does not differ between the cohorts. ${ }^{*} p=0.009$ for IL-10 and ${ }^{*} p=0.04$ for TNF $\alpha$

This article is protected by copyright. All rights reserved 


\begin{tabular}{|c|c|c|c|c|c|c|c|c|c|c|c|}
\hline IL-10 & & & & & Controls & & & Cases & & & \\
\hline Reference & Groups compared & Controls/Losses & Blood drawn & Gestational age & Mean & Median & Range & Mean & Median & Range & Method \\
\hline Jenkins et al 2000 & $\begin{array}{l}\text { Healthy women- } \\
\text { Normal outcome vs. loss }\end{array}$ & $10 / 10$ & Before loss & $5-10$ & $121 \pm 38$ (SD) & - & - & $102 \pm 54$ (SD) & - & - & ELISA \\
\hline Jenkins et al $2000 *$ & $\begin{array}{l}\text { RPL patients- } \\
\text { Normal outcome vs. loss }\end{array}$ & $5 / 5$ & Before loss & $5-10$ & 175 & - & $80-340$ & 55 & - & $30-80$ & ELISA \\
\hline Barrientos et al 2009 * & $\begin{array}{l}\text { Healthy women- } \\
\text { Normal outcome vs. loss }\end{array}$ & $42 / 42$ & Before loss & $4-12$ & $11 \pm 15$ (SEM) & - & - & $5 \pm 6$ (SEM) & - & - & ELISA \\
\hline Calleja-Agius et al 2011-1 *+ & $\begin{array}{l}1^{\text {st }} \text { trimester bleeding - } \\
\text { Normal outcome vs. loss }\end{array}$ & $27 / 53$ & Before loss & $7-10$ & 4.5 & - & - & 1.5 & - & - & Multiplex \\
\hline Vassiliadis et al 1998 & $\begin{array}{l}\text { Healthy women- } \\
\text { Normal outcome vs. loss }\end{array}$ & $19 / 19$ & After loss & $<13$ & $770 \pm 70$ (SEM) & - & - & $860 \pm 60$ (SEM) & - & - & ELISA \\
\hline Paradisi et al $2000 *$ & $\begin{array}{l}\text { Healthy women- } \\
\text { Normal outcome vs. loss }\end{array}$ & $14 / 14$ & After loss & 8-11 & $4.4 \pm 1.4$ (SD) & - & $2-7.5$ & $2.0 \pm 1.1(\mathrm{SD})$ & - & $1-5$ & ELISA \\
\hline Calleja-Agius et al $2011-2^{*}++$ & $\begin{array}{l}\text { Healthy women- } \\
\text { Normal outcome vs. loss }\end{array}$ & $8 / 11$ & After loss & $<13$ & - & 4.5 & $0-15$ & - & 3.5 & $0-12.5$ & Multiplex \\
\hline Verma et al $2018 * * *$ & $\begin{array}{l}\text { Healthy women- } \\
\text { Normal outcome vs. loss }\end{array}$ & $20 / 20$ & After loss & $7-12$ & $16.56 \pm 2.015(\mathrm{SD})$ & - & 6-33 & $17.41 \pm 1.14(S D)$ & - & $10-34$ & Multiplex \\
\hline TNF $\alpha$ & & & & & Controls & & & Cases & & & \\
\hline Reference & Groups compared & Controls/Losses & Blood drawn & Gestational age & Mean & Median & Range & Mean & Median & Range & Method \\
\hline Barrientos et al $2009^{*}$ & $\begin{array}{l}\text { Healthy women- } \\
\text { Normal outcome vs. loss }\end{array}$ & $42 / 42$ & Before loss & $4-12$ & $18 \pm 18$ (SEM) & - & - & $18 \pm 15$ (SEM) & - & - & ELISA \\
\hline Calleja-Agius et al 2011-1 $*_{+}$ & $\begin{array}{l}1^{\text {st }} \text { trimester bleeding - } \\
\text { Normal outcome vs. loss }\end{array}$ & $27 / 53$ & Before loss & $7-10$ & 2.5 & - & - & 1.5 & - & - & Multiplex \\
\hline Freis et al 2018 & $\begin{array}{l}\text { IVF patients- } \\
\text { Normal outcome vs. loss }\end{array}$ & $22 / 17$ & Before loss & 5 & $5.00 \pm 0.26$ (SEM) & - & - & $5.11 \pm 0.40$ & - & - & Multiplex \\
\hline Calleja-Agius et al 2011-2 * & $\begin{array}{l}\text { Healthy women- } \\
\text { Normal outcome vs. loss }\end{array}$ & $8 / 11$ & After loss & $<13$ & - & 4.5 & $0.5-6.5$ & - & 0.5 & $0.5-13$ & Multiplex \\
\hline Verma et al 2018 & Healthy women- & $20 / 20$ & After loss & $7-12$ & $91.84 \pm 16.01$ (SD) & - & $10-320$ & $127.0 \pm 9.19$ & - & $70-230$ & Multiplex \\
\hline
\end{tabular}

This article is protected by copyright. All rights reserved 
Normal outcome vs. loss

* These studies only displayed values graphically, values estimated from figures

+ This study reported the mean TNFa:IL-10 ratio for normal pregnancy (1) and women who miscarried later (4)

++ This study reports an elevated TNFa:IL-10 ratio in pregnancies that had miscarried, no value given

This article is protected by copyright. All rights reserved 


\section{Table 2. Patient demographics}

\begin{tabular}{|c|c|c|c|c|}
\hline & \multicolumn{2}{|c|}{ IVF } & \multicolumn{2}{|c|}{ Natural conceptions } \\
\hline & $\begin{array}{l}\text { Controls } \\
(n=22)\end{array}$ & $\begin{array}{l}\text { Losses } \\
(n=18)\end{array}$ & $\begin{array}{l}\text { Controls } \\
(n=75)\end{array}$ & $\begin{array}{l}\text { Losses } \\
(n=27)\end{array}$ \\
\hline Age (mean $\pm S D)^{*}$ & $35.1 \pm 4.4$ & $37.1 \pm 4.2$ & $28.1 \pm 3.3$ & $30.5 \pm 4.7$ \\
\hline $\mathrm{BMI}($ mean $\pm \mathrm{SD})$ & $25.2 \pm 4.9$ & $25.6 \pm 3.2$ & $24.2 \pm 4.5$ & $25.6 \pm 5.6$ \\
\hline Previous live birth $\mathrm{n}(\%)$ & $7(31.8)$ & $2(11.1)$ & $28(37.3)$ & $12(44)$ \\
\hline $\begin{array}{l}\text { Previous miscarriage } \\
\mathrm{n}(\%)\end{array}$ & $4(18.2)$ & 2(11.1) & $9(12.0)$ & $6(22)$ \\
\hline Caucasian & $73 \%$ & $78 \%$ & $100 \%$ & $100 \%$ \\
\hline
\end{tabular}

*The natural conception cohort was younger, $p<0.01$. 
Table 3. Levels of IL-10, TNF $\alpha$ and their ratio shown at the different biological phases of placentation.

\begin{tabular}{|c|c|c|c|c|c|c|c|}
\hline & & \multicolumn{3}{|c|}{ Controls } & \multicolumn{3}{|c|}{ Losses } \\
\hline & & Peri-implantation & Histiotrophic phase & Perfusion phase & Peri-implantation & Histiotrophic phase & Perfusion phase \\
\hline & & 4-5 weeks & 6-8 weeks & 9-13 weeks & 4-5 weeks & 6-8 weeks & 9-13 weeks \\
\hline & & $N=123$ & $\mathrm{~N}=110$ & $N=120$ & $\mathrm{~N}=93$ & $\mathrm{~N}=42$ & $\mathrm{~N}=15$ \\
\hline \multirow[t]{3}{*}{$\mathrm{IL}-10(\mathrm{pg} / \mathrm{ml})$} & Mean $\pm S D$ & $2.53 \pm 1.28$ & $2.73 \pm 0.84$ & $2.82 \pm 1.10$ & $2.35 \pm 0.80$ & $2.34 \pm 0.77$ & $2.47 \pm 0.85$ \\
\hline & Median & 2.31 & 2.56 & 2.54 & 2.28 & 2.18 & 2.11 \\
\hline & Range & $0.75-10.61$ & $1.53-6.68$ & $1.41-9.85$ & $0.66-5.15$ & $1.03-5.16$ & $1.60-4.73$ \\
\hline \multirow[t]{3}{*}{$\mathrm{TNF} \alpha(\mathrm{pg} / \mathrm{ml})$} & Mean $\pm S D$ & $5.76 \pm 177$ & $5.41 \pm 1.42$ & $5.46 \pm 1.49$ & $6.60 \pm 2.00$ & $6.23 \pm 1.46$ & $5.56 \pm 1.57$ \\
\hline & Median & 5.56 & 5.14 & 5.41 & 6.40 & 6.10 & 5.63 \\
\hline & Range & $1.51-11.33$ & $1.86-10.01$ & $2.36-11.18$ & $2.24-11.23$ & $2.53-9.52$ & $2.41-8.40$ \\
\hline \multirow[t]{3}{*}{ IL-10:TNF $\alpha$ (ratio) } & Mean $\pm S D$ & $0.45 \pm 0.19$ & $0.53 \pm 0.19$ & $0.53 \pm 0.20$ & $0.37 \pm 0.11$ & $0.38 \pm 0.12$ & $0.48 \pm 0.21$ \\
\hline & Median & 0.41 & 0.50 & 0.51 & 0.36 & 0.36 & 0.40 \\
\hline & Range & $0.15-1.16$ & $0.18-1.22$ & $0.19-1.69$ & $0.16-0.63$ & $0.23-0.92$ & $0.29-1.11$ \\
\hline
\end{tabular}

Significant $p$-values can be found in Figure 2 , which shows this data graphically. 
Table 4. Statistical comparison between controls and losses using Cohen's D (p-values). A positive Cohen's D indicates that controls have higher levels than the losses, while a negative Cohen's D indicates the controls have lower levels than the losses.

$\begin{array}{cccc}\text { Pregnancy Week } & \mathrm{IL}-10 & \mathrm{TNF} \boldsymbol{\alpha} & \mathrm{IL}-10: \mathrm{TNF} \boldsymbol{\alpha} \\ 4 & 0.02(0.90) & -0.32(0.10) & 0.26(0.140) \\ 5 & 0.55(0.02) & -0.34(0.15) & 0.93(2 \mathrm{e}-04) \\ 6 & 0.41(0.11) & -0.54(0.07) & 0.80(8 \mathrm{e}-04) \\ 7 & 0.58(0.11) & -0.54(0.14) & 0.87(0.008) \\ 8 & 0.59(0.06) & -0.65(0.04) & 1.00(6 \mathrm{e}-05) \\ 9 & 0.64(0.04) & 0.29(0.52) & 0.40(0.245)\end{array}$



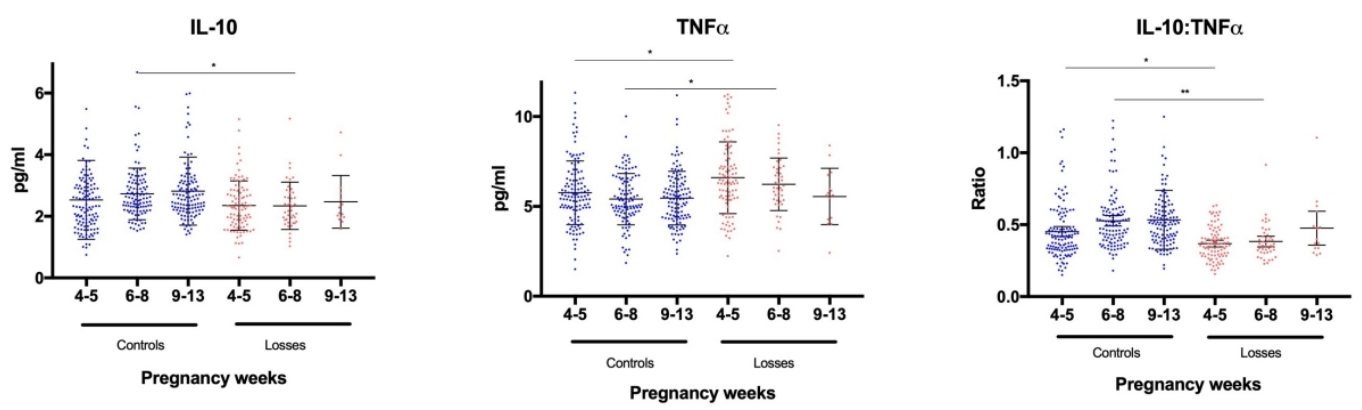

aji_13195_f1.jpg 

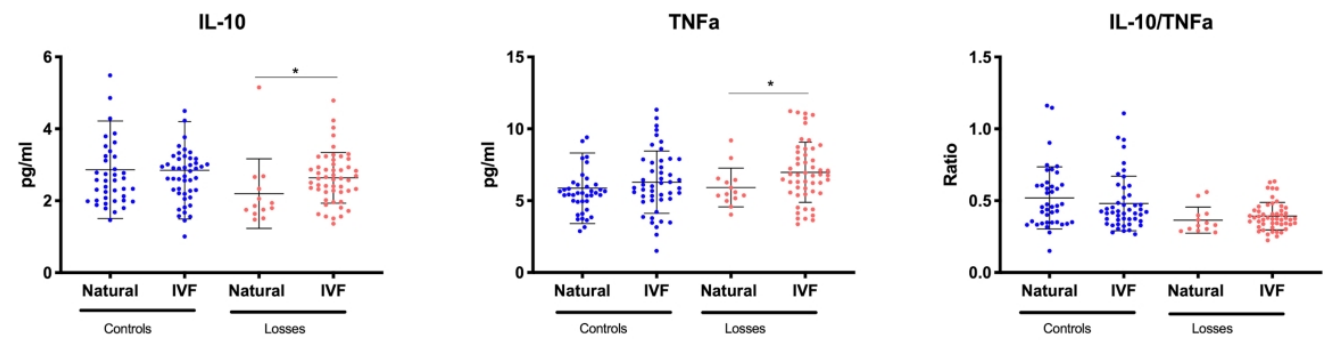

aji_13195_f3.jpg 